\title{
Afectividades en la defensa de los ríos Covento y Sonador (Zona Sur, Costa Rica)
}

Recibido: 25 de septiembre 2017

Revisado: 17 de octubre 2017

Aprobado: 3 de noviembre 2017

Andrés Cambronero

Rodríguez

Costarricense. Licenciado en Psicología de la Universidad de Costa Rica. Ha trabajado en distintas comunidades del país, acompañando organizaciones comunitarias, especialmente en conflictos socioambientales. Está interesado en los temas de psicología social, donde abarca temas políticos, ambientales, agrarios, entre otros.

Correo electrónico: andres.cambronero@proton mail.com

\section{Daniel Fernández Vásquez}

Costarricense. Licenciado en Psicología de la Universidad de Costa Rica. Ha trabajado en diferentes comunidades de este mismo país acompañando organizaciones en conflictos socioambientales, principalmente desde la psicología comunitaria y la educación popular. Algunos temas de interés son: psicología social, política y comunitaria, pedagogías críticas, vínculo

universidad/sociedad y ecología política. Correo electrónico: dan_kero@hotmail.com
Resumen: El presente trabajo profundiza el lugar de las afectividades en los movimientos sociales. Específicamente en la Comisión Defensora de los Ríos Convento y Sonador, que se opuso a la construcción de dos proyectos hidroeléctricos en dichos ríos. Así mismo, se realiza un acercamiento a los efectos psicosociales en las comunidades que se organizaron en contra de estos proyectos. Para lograr lo anterior, se realizaron entrevistas y un taller participativo con las personas de la Comisión. Se puede concluir que la relación que mantienen con los ríos es el núcleo afectivo de la defensa. Además, entre las consecuencias psicosociales se encuentran la generación de conflictos y ruptura de las relaciones en las comunidades.

Palabras clave: afectividades; movimientos sociales; proyectos hidroeléctricos; relaciones comunitarias

\section{Affectivity in the defense of Convento and Sonador rivers (South Region, Costa Rica)}

Abstract: The present work deepens the place of affectivities in social movements, specifically in the case of Comisión Defensora de los Ríos Convento $y$ Sonador which opposed to the building of two hydroelectric projects in these same rivers. In this way, an approach to the psychosocial effects in the communities that organized themselves against these projects is carried out. In order to accomplish these objectives, interviews and a participatory workshop took place with the members of the Comision. It can be seen how the relationship they keep with the rivers is the affective core of their defense. Besides, among the psychosocial consequences, conflict generation and disruption of community relations were identified.

Key words: affectivities; social movements; hydroelectric projects; community relations 


\section{Introducción}

El presente artículo se desprende de la tesis Vivencia y manejo de la afectividad en movimientos sociales: la experiencia de la Comisión de los Ríos Convento y Sonador (Cambronero y Fernández, 2017), que fue realizada por los autores para optar por el grado de Licenciatura en Psicología de la Universidad de Costa Rica. De esta, se tomarán principalmente los vínculos que tienen las personas de la Comisión con los ríos y lo que ello implica en un contexto de conflicto por el agua, en el cual existe la amenaza de la construcción de proyectos hidroeléctricos.

Así mismo, se prevé describir algunos de los impactos en las relaciones comunitarias a causa de la posible llegada de un proyecto hidroeléctrico y la organización que se realizó para detenerlo.

El recorrido de este trabajo será una contextualización del conflicto, el papel de las afectividades en los movimientos sociales, las preguntas y la metodología utilizada, dos apartados con algunos resultados obtenidos y, finalmente, algunas reflexiones en cuanto a lo planteado.

\section{Contextualización del conflicto}

A lo largo de la región latinoamericana, se pueden hallar diversos movimientos que están enfocados en la defensa del agua (Malvares, 2013). En Centroamérica, diferentes procesos económicos y políticos promueven la extracción desmedida de recursos naturales, en los que se incluyen tanto la explotación de la tierra como de las personas. Entre esos procesos podemos identificar el llamado Plan Puebla Panamá, que más tarde modificaría su nombre a Proyecto Mesoamérica. Por medio de este se pretende integrar económicamente toda la región, en aras de abrir, entre otros esctores, el mercado eléctrico. Dicha apertura se ha materializada por medio del Sistema de Integración Eléctrica de Países de América Central (Comisión Defensora de los Ríos Convento y Sonador, 2016).

La apertura de este mercado ha hecho que los inversores no se hagan esperar, generando diversas iniciativas para la producción eléctrica privada en todos los países del istmo centroamericano, sobre todo, proyectos hidroeléctricos (Otros Mundos AC, s.f.). Los anteriores cuentan con financiamiento directo o indirecto por parte de organismos internacionales como el Banco Mundial, el Fondo Monetario Internacional (FMI) y el Banco Centroamericano de Integración Económica (BCIE), los cuales promueven dinámicas extractivistas que finalmente acarrean consecuencias socioambientales irreparables (Comisión Defensora de los Ríos Convento y Sonador, 2016).

En Costa Rica existen 23 represas hidroeléctricas públicas y 38 privadas. El mercado eléctrico, acompañado de una legislación que lo promueve (Leyes 
n. ${ }^{\circ} 7200$ y n. ${ }^{\circ} 7508$ principalmente) impulsa la explotación de los ríos que aún no han sido utilizados para la generación de electricidad (Comisión Defensora de los Ríos Convento y Sonador, 2016). Sin embargo, diferentes comunidades se han organizado para defender los ríos y evitar la construcción de este tipo de proyectos.

Al menos desde 1995, según consta en una nota de la Federación Ecologista del 9 de agosto del 2015, las comunidades cabécares defendieron el río Pacuare para que no se construyera un proyecto hidroeléctrico. El movimiento se mantuvo y creció hasta que el 28 de agosto del 2005 se celebró un plebiscito en Turrialba: $97 \%$ de la población se opuso a la construcción de proyectos hidroeléctricos en el Pacuare (Federación Ecologista, 2015).

Igualmente, por la presión de comunidades organizadas, en el 2014 la municipalidad de Coto Brus firmó un acuerdo que generaba una moratoria para detener todos los proyectos hidroeléctricos del cantón, hasta que se formalizaran los consentimientos libres, previos e informados en las comunidades (Federación Ecologista, 2014). Luego de esa moratoria, la municipalidad de Upala el 13 de febrero del 2015 hizo lo mismo: detuvo por cinco años "el otorgamiento de cualquier autorización municipal que se requiera para la instalación de proyectos hidroeléctricos en el cantón, mientras se elaboran estudios del impacto social y ambiental acumulativo de todas las represas ya autorizadas en el municipio" (Federación Ecologista, 2015). En aquel momento, existían tres proyectos en funcionamiento y siete en trámite. Cabe destacar que, ante lo sucedido por el huracán Otto en Upala a finales del 2016, las comunidades solicitaron que se investiguen los proyectos hidroeléctricos de Bijagua y Canalete, a fin de reconocer si de alguna manera tuvieron responsabilidad del desastre (Madrigal y Gómez, 2017).

Entre el 2012 y el 2014, 16 proyectos hidroeléctricos se presentaron ante la Secretaría Técnica Nacional Ambiental (SETENA) para recibir la viabilidad ambiental por parte de distintas empresas privadas (Federación Ecologista, 2017). Ante dicha situación, en la Zona Sur de Costa Rica, se organizó el Movimiento Ríos Vivos, el cual es conformado por quince organizaciones comunitarias de los cantones de Pérez Zeledón, Buenos Aires y Coto Brus (Comisión Defensora de los Ríos Convento y Sonador, 2016). Gracias a la lucha que han realizado estas comunidades y el apoyo de Ríos Vivos, se han archivado nueve de los 16 proyectos (Federación Ecologista, 2017), contando Monteverde I y II, los cuales amenazaban los ríos Convento y Sonador.

La Comisión Defensora de los Ríos Convento y Sonador es uno de estos grupos que se han organizado para defender los ríos y que llegan a conformar el movimiento Ríos Vivos. Surgió en el 2013, en las comunidades de Longo Mai, Convento y Cristo Rey, en la Zona Sur de Costa Rica. En palabras de ellos mismos, su "grupo nace [sic] como respuesta a la construcción de dos proyectos hidroeléctricos en los ríos que atraviesan nuestras comunidades. Tras [sic] un proceso de tres años de lucha y gracias a la presión y las gestiones realizadas, logramos que ambos proyectos fueran archivados" (Comisión Defensora de los Ríos Convento y Sonador, 2016, p. 10). 
En la dinámica de la Comisión, parten del trabajo comunitario para resistir y construir, gestando lazos de solidaridad y vinculaciones con otras organizaciones en la búsqueda de la defensa de la vida (Comisión Defensora de los Ríos Convento y Sonador, 2016). Para mediados y finales del 2015, se presentaron como uno de los grupos mejor organizados y sólidos de la región sur del país. La capacidad para enfrentar autoridades, de reaccionar ante eventualidades, de movilizar personas, de generar recursos, de visitar otras comunidades, así como la relación con organizaciones de otras zonas del país, la hacen tener un carácter especial entre los demás.

Al realizar el trabajo del que surge este escrito, la Comisión estaba conformada, principalmente, por ocho personas -dos mujeres y seis hombres- quienes cuentan con las características de haber participado y haberse comprometido con la lucha de la Comisión a lo largo del proceso. Estas personas asistieron y colaboraron en la organización de talleres, reuniones, caminatas y actividades.

La defensa de los ríos que llevó a cabo la Comisión estuvo permeada por diversas tensiones que surgían ante el enfrentamiento contra empresarios, vecinos, vecinas, e incluso con las instituciones estatales que parecían estar favoreciendo los proyectos hidroélectricos. Dichas tensiones generaron variados afectos, que podían pasar desde hacer más fuerte su compromiso con la lucha y la indignación ante el otorgamiento de permisos por parte del gobierno a las empresas, hasta el rompimiento de vínculos con personas que participaban en la Comisión, entre otros.

También existieron otros afectos que emergieron en el proceso de las vinculaciones, decisiones y acciones que realizaron. Lo anterior hace necesario el reconocimiento y la validación de la función de las afectividades en los movimientos sociales, que es el objetivo de este trabajo. Para ello, se toma como caso específico la Comisión Defensora de los ríos Convento y Sonador, ubicada en el cantón de Buenos Aires de Puntarenas, Costa Rica.

\section{Afectividades en movimientos sociales}

Las afectividades presentan la característica de ser resistentes e incompatibles con las conceptualizaciones que no tienen posibilidad de transformación y movimiento. Su origen depende del contexto en el que se encuentra, el cual, particularmente, nunca es el mismo. Si se creara una categoría, posiblemente al instante siguiente se quedaría vacía y las afectividades aparecieran en otro lugar porque estas sorprenden y aparecen sin formas predeterminadas que dan la posibilidad de otras configuraciones y que permiten la creación, la demovilización, la acción o la misma parálisis.

Al hablar de las afectividades, se está haciendo referencia de igual forma a las pasiones, las emociones o las sensaciones que en palabras de Fernández (2000, p.25) "son todos sentimientos, y viceversa en cualquiera de sus combinatorias: todos son todos". Esto lleva a afirmar que en la cotidianidad 
no hay diferencia entre las unas y las otras, y son utilizadas para referirse a algo que nos atraviesa, que nos afecta y que escapa a estrictas clasificaciones.

Las afectividades serían, entonces, todo el conjunto de lo que se siente o emociona, y que se pueden encontrar en una infinidad de formas como el amor, la solidaridad, el miedo, el rechazo, el orgullo y las combinaciones que se puedan generar entre ellas y muchas otras que a veces no se pueden nombrar. Además, tienen el carácter de ser políticas porque con ellas se potencian las acciones, las resistencias y los movimientos sociales; o, por el contrario, las pueden desarticular.

El enojo -para las personas de la Comisión- emerge cuando se quieren llevar sus ríos y eso les moviliza a defender; se alegran cuando los proyectos hidroeléctricos se van, y hacen una fiesta; y en el transcurso de los procesos de los movimientos sociales, personas salen y otras ingresan, por diferencias, acuerdos, desacuerdos, chismes, entre otras razones. Están ahí, en el ser político, en la lucha, haciendo caer en la desesperanza o creando las utopías más anheladas que impulsan a caminar.

Desde esa línea, al considerar el papel político y movilizador de las afectividades se encuentra una coincidencia con lo planteado por Jasper (2012), quien considera que cualquier forma de explicación sobre la vinculación en movimientos sociales (desde identidades, narrativas o frames) estaría incompleta si se deja por fuera el factor emocional. Por esta razón, identificar y analizar las afectividades presentes en el trabajo de la Comisión es fundamental para comprender la vinculación de sus integrantes en esta.

El mismo Jasper (2012) menciona una serie de estudios que se han realizado desde inicios de los noventa, en los cuales se demostró la significativa relación entre diferentes emociones -ira, enojo, indignación, esperanza, solidaridad- y las metas de las personas al vincularse en protestas o movimientos sociales. Entre estas metas se pueden mencionar los objetivos políticos, la reputación, la sensualidad, la radicalización, los medios de acción, la manifestación retórica y la dinámica grupal.

Además, otros autores (Stekelemburg y Klandermans, 2010; Stürmer y Simon, 2009; Sabucedo, Durán, Alzate y Barreto, 2011) coinciden con lo anterior: plantean que emociones como el miedo y la indignación, entre otras, están imbricadas en las formas de vinculación de las personas con los diversos movimientos sociales. Estos últimos se van a comprender, a partir de definiciones de otros autores como Tarrow (1994), Zibechi (2003), Touraine (2006) y Martín Baró (1989), como:

Aquellos grupos en los cuales sus miembros se han identificado a partir de emociones, ideologías y quejas compartidas, creando una vinculación afectiva que surge del rechazo a identidades oprimidas, y que tienen como obje- 
tivo otra sociedad libre de la opresión, pobreza e injusticia, con mayor autonomía, y que para esto se mantienen permanentemente promulgando su actitud de una sociedad liberada. (Cambronero y Fernández, 2017, p. 30).

Adicionalmente, Fernández (2000) realizó un trabajo en el que retomó a autores como Morin y Le Bon. Su investigación permite describir las afectividades como formas complejas que emergen de las relaciones entre las personas y en el contexto que habitan. Las afectividades estarán en constante transformación, variando con el conjunto de relaciones. $Y$ aunque las emociones se viven y se expresan de manera individual, se comparten culturalmente.

De ese modo, es posible conjugar las afectividades y los movimientos sociales, en el sentido de que en estos últimos existen procesos relacionales que hacen emerger diversas emociones. Hernández (2016) ha retomado el concepto de afectación para describir cómo las personas se identifican a partir de quejas, problemas en común, y, sobre todo, por una sensibilización, que les hace organizarse y movilizarse para defender sus intereses. La autora agrega lo siguiente:

La insurgencia de las multitudes es sobre todo un fenómeno psíquico y afectivo. El sentirse afectado o implicado en algo, no es sólo un elemento que tiene que ver con una cuestión de condiciones objetivas como el hambre o la pobreza, sino que se trata de un desplazamiento de la sensibilidad, en donde lo que antes parecía normal e incuestionable, se convierte en un grito que no es posible sostener (p. 169).

Entonces, reconocemos los movimientos sociales como formas afectivas en las que sus integrantes han sido afectados y al mismo tiempo han afectado a otras personas, y desde las emociones que les habitan conjugan acciones, objetivos y tareas para defender sus intereses.

\section{Una aproximación a las afectividades en la vivencia de las personas de la Comisión}

Teniendo en cuenta la relevancia de las afectividades en los movimientos sociales, se decidió indagar en cómo la relación con los ríos afectó el involucramiento y la participación de las personas de la Comisión en su proceso de resistencia. También interesa contestar cómo fueron afectadas las relaciones 
con las comunidades ante la llegada de los proyectos hidroeléctricos y la organización de la Comisión.

Para abarcar estas preguntas desde las mismas personas de la Comisión, se realizaron dos entrevistas a cada una, en las que se profundizó tanto en sus biografías como en el proceso que vivieron durante la defensa de los ríos Convento y Sonador. También, en un taller grupal, se trabajaron los significados y los sentires de las personas participantes con respecto a tales ríos.

El contenido que emergió de las entrevistas y del taller en relación con las preguntas planteadas, se presenta en los siguientes dos apartados.

\section{La relación con los ríos: el núcleo afectivo para la defensa}

Partiendo de los afectos para entender la relación entre las personas de la Comisión con los Ríos Convento y Sonador, es posible dar cuenta de lo que sienten y de sus razones afectivas para defenderlos. "Los ríos siempre han estado" (comunicación personal, Jiri, 2016), mencionaba uno de los participantes al hablar sobre el lugar que han tenido estos en su vida. Se puede reconocer cómo en sus historias personales, sus relaciones familiares y hasta en sus creencias espirituales se pueden hallar sentires que los han llevado a posicionarse en la defensa de los ríos.

En las entrevistas se pudo notar que las personas de la Comisión tienen una relación afectiva con los ríos. Esta se gesta y se mueve en distintos lugares y momentos que han vivido, y puede estar representada tanto en historias, así como en las intenciones que ellas y ellos relatan con respecto a la forma en que los ven y los viven. Si bien esta relación se expresa de una forma única para cada integrante de la Comisión, es posible encontrar puntos de encuentro a partir de los cuales lograron organizarse para defenderlos.

Desde su niñez, la relación con la naturaleza ha sido cercana. Ya fuera porque de esta dependiera su alimentación; o porque sus cotidianidades eran vividas rodeados por la montaña y los ríos, o por ambas. Los ríos han formado y forman parte de sus vidas porque su comida, su trabajo, su cotidianidad y hasta su misma identidad son posibilitadas por el agua que los recorre, como podemos ver en el siguiente extracto de una de las entrevistas:

Di, la gente lo tenía para riego de todos los cultivos. Digamos, desde eso se utiliza para los cultivos, y se traía la tubería que había para consumo era del río porque no se tenía de las nacientes; toda el agua se traía del río (comunicación personal, Ernesto, 2016).

El río no es concebido como un mero recurso, sino que está presente en sus cotidianidades, haciendo posible su alimentación, cultivos, higiene, entre 
otras necesidades básicas para vivir. Otras personas, consideran a los ríos como "amigos" o como parte de la "madre naturaleza", que provee y que se cuida. Tanto así que verlos destruidos dolería más que el daño a sí mismas:

Yo baño [sic] veinte años en este río, y después quieren secarlo. Me siento es como, es un amigo mío personal. Yo me siento como... alguien quiere matar mi amigo. Entonces no me gusta meterme porque puede matar a mí también a la vez, pero no puedo dejarlo solo porque me disgustaría yo mismo a mí mismo. Tanto que sufriría más que si me matan (comunicación personal, Cristoph, 2016).

Además, en ocasiones son sentidos incluso como una parte del mismo cuerpo, como mencionó una de las integrantes de la Comisión:

El río, siempre era como el río, o la esperanza de que el río fuera libre; o sea, es que yo siento que ese era el objetivo porque no siento que el río era un objeto o así, el río es parte de mí, entonces como ¿qué me motiva? di, que no me corten la mano, ¿qué me motiva? Que no me corten el pie (comunicación personal, Yendry, 2016).

Se reconoce también cómo la relación con los ríos conlleva un cuidado recíproco. Se hace uso del agua y esta "da vida"; por lo tanto, se le debe proteger y cuidar. Es desde ahí que se puede ver cómo la defensa de los ríos no es solamente una defensa de la naturaleza per se, sino que significa defender su alimentación, su trabajo y hasta su misma identidad. Es, al fin y al cabo, defenderse a sí mismas y a sí mismos, como mencionaba uno de los integrantes:

Diay, es que cuando las cosas se ven que se inician a matarlo a uno o a quitarle la vida a uno, como el agua que es la vida de todo ser humano, diay, nos quitan el agua que, ¿qué están haciendo con uno? Lo están matando (comunicación personal, Ernesto, 2016).

Al reconocer lo anterior, no extraña que, ante el ataque a los ríos surjan la indignación y la rabia; pero también la esperanza ante la defensa de la vida. Afectos que, como menciona Fernández (1994), buscan mantener y crear di- 
ferentes vínculos cuando se sienten amenazados. El intento de represar los ríos para convertirlos en una corriente eléctrica hizo a las personas de la Comisión hallarse ante la posibilidad de que el lugar de vida que han conocido junto a estos y sus estilos de vida fueran aniquilados para el beneficio económico de otras personas, y eso no era aceptable.

Así, se puede señalar que la defensa de los ríos Convento y Sonador tiene un núcleo afectivo que es la relación de las personas de la Comisión con ambos ríos. Esta es alimentada, como las entrevistas lo demuestran, por sus historias de vida, sus relaciones familiares, por su concepción y relación con la naturaleza y por el mismo vínculo con los ríos, lo que los ha llevado a luchar, a defender algo que quieren o aman:

Pues para mí, eso me hace sentirme bien pues se lucha por lo que usted quiere, ¿me entiende? Porque si usted va a luchar por algo que no quiere, al final no lo va a sentir. Pero si usted aprecia aquello, usted quiere aquello, entonces usted se siente bien porque usted está luchando por lo que usted quiere (comunicación personal, Carmen, 2016).

\section{La organización de la Comisión y los efectos en las comunidades}

Al iniciar este proceso de defensa no todas las personas que habitaban las comunidades que son atravesadas por los ríos Convento y Sonador se oponían a la construcción de los proyectos hidroeléctricos. El discurso con el cual se realizaron las propuestas de estos a la comunidad incluía promesas de "desarrollo" y trabajo. Estas en ocasiones promocionadas por líderes de iglesias en las comunidades, lo cual tenía bastante peso entre muchas de las personas de la comunidad y el hecho de oponerse también tenía sus consecuencias:

Hasta en la iglesia estuvo promocionando la hidroeléctri-

ca. Era el delegado; era el presidente; entonces, él pedía que lo apoyaran ¿verdad?, porque iban a pavimentar el camino y que iban a arreglar la escuela, y que el salón comunal estaba cayéndose y que era la oportunidad de arreglarlo y de hacerlo nuevo. Entonces, todo eso era la motivación; entonces, al ir en contra, ya eran enemigos míos. Yo me topaba al cuñado, hablando con el tío que 
era también fontanero y pasaba y saludaba al tío y a mí no me saludaba; me topaba a ciertas personas y no me saludaban. Yo era ya una espina para muchos (comunicación personal, Beltrán, 2016).

Lo anterior llevó a que muchas personas de las comunidades estuvieran en favor e incluso defendieran los proyectos hidroeléctricos. En algunos casos, estas llegaron a dar sus firmas y a vender terrenos para que los empresarios llevaran a cabo el proyecto. Tales situaciones provocaban sentires como enojo y tristeza entre las personas de la Comisión, emociones que venían del reconocimiento del engaño por parte de los empresarios, y de la crítica a la falta de protección de otras vecinas y otros vecinos hacia los ríos:

Lo que el sentimiento que no era agradable, era que los vecinos hayan vendido o negociado los derechos de paso para el proyecto hidroeléctrico, siendo de la comunidad. Yo nunca, ninguno de esos era amigo mío; entonces, nunca, ajá, pero otro miembro de la Comisión sí conocía alguno de esos; y sí, y discutió mucho de eso con ellos (comunicación personal, Jiri, 2016).

Estas diferencias, como se puede observar en las dos citas anteriores, llevaron en ocasiones a discusiones y tensiones dentro de la comunidad, en relaciones que previo al intento de construcción de los proyectos hidroeléctricos mantenían una cierta tranquilidad.

Adicionalmente a tales conflictos, diferentes personas de la Comisión mencionaron que en más de una ocasión fueron señaladas como vagabundas y vagabundos por el trabajo que realizaban. Que además de que no se trataba de una labor remunerada, requería una constante sensibilización hacia las personas de las comunidades; por lo tanto, en ocasiones eran comparados con personas del gobierno "que no hacían nada" o que buscaban un beneficio personal, como menciona Ernesto en su entrevista:

Yo supe lo que fue llegar a algunas casas dentro de los mismos pueblos. Algunos me decían: "Ya te convertiste en un vago", "A estas horas andas vagueando", “¿Qué es lo que andas buscando?" o “¿Sos un funcionario del Gobierno, que traes tantos papeles?" (comunicación personal, Ernesto, 2016). 
De esta manera, se iban generando roces entre las personas de la Comisión y de las comunidades, lo cual llegó a afectar relaciones de muchos años: distintos intereses se habían creado alrededor de los proyectos hidroeléctricos. Así lo demuestra el siguiente comentario:

Entonces, era doloroso escuchar todas esas cosas todo el tiempo y empezar a ver lo duro, digamos, que fue el ver que una persona era amiga de uno y de repente no era amiga de uno. No se sabía quién era enemigo de uno en ese momento. $\mathrm{Y}$ el miedo de alguna manera interno que uno tiene, de que puede ser afectado, que le pueden hacer algo. Porque al usted ser una persona, aunque yo traté en todo momento de escaparme, diay no podía dejar la lucha; tenía que seguir; era una responsabilidad, una obligación (comunicación personal, Beltrán, 2016).

Así se puede reconocer cómo la amenaza de los ríos en su forma de construcción de proyectos hidroeléctricos no solamente movió afectividades en las personas de la comisión. También se hizo presente en las relaciones comunitarias y, por lo tanto, la lucha y defensa debieron pasar por ahí. A pesar de los conflictos que se estaban generando, las personas de la Comisión en su trabajo mantuvieron presente la importancia de informar a las comunidades.

Reconocían que quienes no estaban apoyándoles, en su mayoría lo hacían por falta de información y no precisamente porque no les importaban los ríos. Además, afirmaban que su trabajo buscaba el bien para todos, incluso para quienes no estaban de su lado.

A partir de esto decidieron trabajar junto a otras personas para informar a las comunidades. La consecuencia fue que, al avanzar la lucha, la mayoría de las comunidades los apoyaron, si bien no necesariamente asistiendo a las reuniones, sí participaban en las actividades; o firmaban los documentos para las oposiciones escritas que eran vitales para el proceso de defensa. Este proceso motivó a las personas de la Comisión, quienes pasaron de ser rechazadas a sentirse acompañadas:

Súper bien porque uno ya sabía que no estaba solo; sa-

bía que esas personas si no iban a acuerparse con uno en una protesta, por lo menos estaban con su nombre y con su firma; y entonces, ya eso lo hacía sentirse a uno que ya le iban a poner más cuidado. En cualquier lugar 
que llegara usted y llegara con sesenta o setenta firmas o cien firmas, ya era diferente a que llegaran cuatro pelados como éramos nosotros, a manos cruzadas ahí, a decir que nos oponíamos (comunicación personal, Luis, 2016).

De esta manera, se puede reconocer cómo la carga afectiva que suponía mantenerse contrario a los que fueron sus amistades de infancia y vecinos y vecinas de toda la vida jugó su papel para decidir mantenerse. Las personas de la Comisión reconocían los engaños que les hacían a sus amigos, amigas y familiares, y, por eso, "no aflojaban". Continuaron informando y acercándose de distintas maneras para que las personas se dieran cuenta de los efectos negativos que les traerían esos proyectos.

Igualmente, es posible determinar cómo las relaciones con las comunidades fueron fundamentales en la defensa de los ríos Convento y Sonador. Además, es notable cómo las afectividades de las personas de la Comisión fueron constantemente movilizadas por las relaciones que se gestaban en las comunidades, las cuales pasaron por la incomodidad, la angustia y en algunos momentos hasta por el miedo de perder relaciones de amistad de años. Sensaciones que, a partir del trabajo y la sensibilización, lograron convertirse en motivación y alegría por el apoyo que llegaron a recibir desde las comunidades.

\section{Reflexiones finales}

Las personas involucradas en la Comisión Defensora de los Ríos Convento y Sonador fueron afectadas y afectaron a otros desde el momento en que recibieron la noticia de la amenaza de los proyectos hidroeléctricos hasta cuando lograron archivarlos. El proceso para que esto último ocurriera, conllevó una serie de estrategias, objetivos y alianzas que tomaban parte de sus vidas, convencidas de que era lo que debían hacer. No se imaginaban sin poner sus cuerpos, sin hacer la lucha o sin asistir a las reuniones que convocaban para hacer la resistencia. La razón afectiva que les movilizaba, y que posiblemente les sigue movilizando, era, para cada una y cada uno, muy importante: la defensa de dos ríos que son parte de sus vidas.

En el apartado teórico se mencionó que las afectividades son comprendidas en las relaciones, en el "entre" de quienes se relacionan, donde existe la capacidad de afectación. Así se puede reconocer en la vinculación entre las personas de la Comisión y los ríos Convento y Sonador. El haber nacido cerca de estos, en los cuales se jugaba, se visitaban, se iba a buscar alimento, entre otras prácticas cotidianas, les hizo que fueran interiorizados como algo propio. 
Por esta razón, es importante recalcar que en la cotidianidad de las personas emergen las afectividades, en el conjunto de interacciones que se viven y se sienten. De ahí que exista una red de significantes, sentires y simbolismos en las cotidianidades de las personas, relacionadas con los ríos. Y para quienes integran la Comisión, estos se encuentran dentro de su diario vivir desde muy tempranas edades.

Así mismo, han construido sus propias identidades que catalogan como "campesinas". Dentro de las cuales, la relación con los ríos y su cuidado también forman parte de esa autoimagen. Defenderlos va más allá de defender un río, es defender a un amigo, su alimentación; incluso, sus propias vidas. Forman parte de lo que les permite estar, sentir y ser en el mundo.

Ante esto, se puede decir que los ríos Convento y Sonador son el núcleo afectivo de las personas de la Comisión, y las moviliza a defenderlos. Verlos amenazados puede ser la causa de del enojo y la razón de los deseos de archivar los proyectos hidroeléctricos. Esto coincide con los autores como Jasper (2012), que reconocen estos afectos como parte de los generadores de los movimientos sociales. De hecho, se puede agregar que el reconocimiento de las personas de la Comisión sobre el Plan Mesoamérica o Plan Puebla Panamá les permitió comprender su conflicto como parte de un contexto económico global, que en palabras de ellas mismas responden a intereses de unos pocos empresarios. Se generó, pues, más rabia e indignación, que los impulsó a defender y luchar por sus ríos. Es decir, esta toma de conciencia les permite dar un sentido y objetivo político a sus afectividades.

“¿Cómo es posible que se vengan a llevar nuestros ríos?" (Yendry, comunicación personal, 2016), mencionaba una compañera con un tono de indignación y de incomprensión. Sentimientos que en conjunto con los de sus otros compañeros y compañeras se fortalecieron, y finalmente les permitió organizarse, mantenerse y ganar su lucha.

Pero, como se mencionó en los resultados, la llegada de los proyectos hidroeléctricos no solo movilizó a las personas que integran la Comisión, sino también los afectos en las relaciones comunitarias. Los intereses económicos y políticos que prometieron quienes incentivaban esos proyectos, tuvieron como consecuencia cierta polarización en las comunidades, al parecer inexistente anteriormente, que provocó la confrontación entre personas.

Entonces, sucedió que amistades de años e incluso relaciones familiares fueron afectadas, lo cual significó una tristeza para las personas de la Comisión, quienes, además de defender los ríos, debían enfrentar a personas cercanas. Caminar por las comunidades no era lo mismo; sobre todo, en los inicios de la lucha cuando no se reconocía quiénes estaban en favor o en contra de los proyectos hidroeléctricos. Se trataba de personas que meses antes se saludaban con gran cordialidad, pero ahora daban la espalda y les criticaban.

Sin embargo, el trabajo de sensibilización realizado por la Comisión tuvo efectos en el cuidado de las relaciones comunitarias. La ejecución de talleres y caminatas informativas permitió mostrar las consecuencias de los proyectos hidroeléctricos, desmintiendo lo que ellos y ellas nombraban como falsas 
promesas. De esta manera, se aclaraban las dudas en las personas de las comunidades, las tensiones disminuían y se empezaban a reconocer en una misma lucha

A partir de lo anterior, podemos plantear cómo la llegada de proyectos hidroeléctricos y posiblemente de otros similares (como lo serían un aeropuerto, minería, expansión de monocultivos, entre otros) provocan consecuencias ambientales. También tienen un efecto a nivel psicosocial de las comunidades, generando tensiones y conflictos, que pueden llevar a "romper" tejidos comunitarios. Siendo así, es importante cuestionarse sobre las repercusiones que generan estos proyectos, la responsabilidad de los gobiernos y el papel que podrían jugar diferentes ciencias sociales en torno a estas.

Algunas preguntas podrían ser las siguientes: ¿Qué responsabilidades tienen los gobiernos centrales ante estas consecuencias y cómo podría asumirlas?, ¿cómo plantearse un proyecto de trabajo alrededor de las tensiones comunitarias generadas por un conflicto socioambiental?, ¿desde dónde es posible llevar a cabo una reparación del tejido comunitario? y, finalmente, ¿cuáles podrían ser las vías para realizar esta tarea?

En las comunidades, las consecuencias psicosociales a causa de los proyectos hidroeléctricos no requieren específicamente que estos se lleven a cabo. La simple propuesta puede llegar a tener implicaciones. Por ende, las preguntas planteadas en el párrafo precedente pueden llegan a ser válidas tanto en poblaciones donde los proyectos se han llevado a cabo, así como en las que aún se resisten y en las que fue posible defender la vida, deteniendo las amenazas, como es el caso de las comunidades que son recorridas por los ríos Convento y Sonador.

Las afectividades en movimientos sociales continúan siendo un tema por investigar, trabajar y sentir, reconociendo las distintas experiencias de resistencia que existen. Son, como ya mencionó Jasper (2012) y como se desprendió de esta experiencia, parte vital para comprender los movimientos sociales y las experiencias de las personas que se involucran y participan, así como de las comunidades ante las diferentes amenazas y resistencias. Entonces, deben seguirse realizando estudios que indaguen y recalquen qué hacen las personas con los afectos, cómo son manejados, qué implicaciones tienen en sus subjetividades y cómo llevarlos de lo personal a lo político.

\section{Referencias}

Comisión Defensora de los Ríos Convento y Sonador. 2016. «Defensa de los ríos Convento y Sonador. La historia de nuestra lucha». Universidad de Costa Rica, Vicerrectoría de Acción Social Programa Kioscos Socioambientales para la Organización Comunitaria 
Federación Ecologista (2014, mayo 5). «Coto Brus se suma a los cantones libres de hidroeléctricas». Recuperado de http://www.feconcr.org/index.php? option=com_content\&task=view\&id=2349\&Itemid=99999999

Federación Ecologista (9 de agosto, 2015). «Comunidades piden a Presidencia decreto para no represar ríos Pacuare y Savegre». Recuperado de http://www.feconcr.org/index.php? option=com_content\&task=view\&id=2473\&ltemid=99999999

Federación Ecologista (2017, marzo 28) «Archivado noveno proyecto hidroeléctrico en zona sur». Recuperado de http://www.feconcr.org/index.php? option=com_content\&task=view\&id=2624\&ltemid $=76$

Fernández, Pablo. 2000. «La Afectividad Colectiva». México D.F.: Taurus. Fernández, P. (1994). «La Afectividad Colectiva y su Geometría Política». Comportamiento, 3 (2), 100-111.

Madrigal, César; Gomez, Edis. (2017, marzo 24). «Upaleños piden investigación por daños a represa durante el Huracán Otto». Lapresalibre.cr. Recuperado de https://www.laprensalibre.cr/Noticias/detalle/106628/upalenos-pideninvestigacion-por-danos-a-represa-durante-el-huracan-otto

Fernández, Pablo. 2000. «La Afectividad Colectiva». México D.F.: Taurus. Hernández, Tania. 2016. «Formas Afectivas en los Movimientos Sociales». Tesis de maestría. Universidad Autónoma de México. Jasper, James. 2012. «Las emociones y los movimientos sociales: veinte años de teoría e investigación». Revista Latinoamericana de Estudios sobre Cuerpos, Emociones y Sociedad, 4(10): 48-68.

Malvares, Mirta. 2013. «Gestión del agua en el contexto de la globalización. Una aproximación de análisis desde la perspectiva de la sociedad del riesgo». Cambio climático, movimientos sociales, y políticas públicas. Una vinculación necesaria. Santiago: CLACSO. 
Martín-Baró, Ignacio. 1989. «Sistema, grupo y poder. Psicología Social desde Centroamérica Il». San Salvador: UCA Editores.

Otros Mundos AC. s.f. «Del PPP al Proyecto Mesoamérica». Chiapas: Otros Mundos AC.

Sabucedo, José Manuel, Mar Durán, Mónica Alzate y Idaly Barreto. 2011. «Emociones, ideología y acción política colectiva». Universitas Psychologica, 10(1): 27-34.

Stekelemburg, Jaqueline y Bert, Klandermans. 2010. «The social psychology of protest». Sociopedia.isa, pp.1-13

Stürmer, Stefan y Bernd Simon. 2009. «Pathways to Collective Protest: Calculation, Identification, or Emotion? A Critical Analysis of the Role of Group-Based Anger in Social Movement Participation». Journal of Social Issues, 681-705.

Tarrow, Sidney. 1994. «El poder en movimiento: Los movimientos sociales, la acción colectiva y la política». Madrid: Alianza.

Touraine, Alain. 2006. «Los movimientos sociales». Revista Colombiana de Sociología, 27, pp. 255-278.

Zibechi, Raúl. 2003. «Los movimientos sociales latinoamericanos: tendencias y desafíos». Observatorio Social de América Latina. 9(1), pp.185-188. 